\title{
Life Span Changes in Affectivity and Influence of Gender, Health Self-rate, Morbidity, Memory and Mental Stress
}

Aleksandr Kaipov ${ }^{1^{*}}$, Marcos A Sanchez-Gonzalez ${ }^{2}$, Ross W May ${ }^{3}$, Rhaisa Dumenigo ${ }^{1}$ and Juan D Oms ${ }^{1}$

${ }^{1}$ Department of Psychiatry, Larkin Community Hospital, South Miami, Florida, USA

${ }^{2}$ Department of Family Medicine, Larkin Community Hospital, South Miami, Florida, USA

${ }^{3}$ Family Institute, The Florida State University, Tallahassee, Florida, USA

\begin{abstract}
Background: The aim of the present study was (i) examine positive and negative affectivity, its rate of change, gender specificity and (ii) explore the association between health self-rating, number of chronic diseases, memory, and mental stress.

Methods: Data obtained from the Longitudinal Study of Generation was used to assess participants in 3-year intervals from 1985 to 2000 . Positive and negative affect along with health self-rating, morbidity, memory, mental stress were assessed.

Results: A total of 2024 participants (aged 16-99 years; 57\% Female) were considered for analysis. Linear growth models showed that both positive and negative affect decrease over generations. There was no difference between genders for positive affect; negative affect decreased in higher degree in females than in males. Health self-rates increased along the life span with no gender differences. Morbidity increased; memory did not change over generations in both genders. Mental stress didn't change over generations, but was higher in females. Lower health self-rate was associated with higher negative affect and a deeper drop of positive affect later in life. Higher morbidity was associated with lower positive affect. Memory worsening was associated with a decrease of positive affect. Mental stress was a predictive factor for lower positive and higher negative affect. Conclusion: Possible unfavorable influence of decrease of positive affect with life progression is balanced with concomitant decrease of negative affect. Negative affect seems to be more independent from somatic morbidity than positive affect and has higher gender specificity by being lower in females.
\end{abstract}

Keywords: Positive affect; Negative affect; Gender specificity; Health self-rate; Morbidity; Memory; Mental stress

\section{Introduction}

Positive and negative emotions (affectivity) are an important part of individual's physical, mental, and behavioral health. With regard to an individual's lifespan trajectory, previous research suggests that observed and expressed emotions (specifically positive affect) remains relatively stable from young to middle adulthood [1], possibly increasing in adulthood [2-4] and then slightly decreasing in old age [5]. In contrast, negative affect decreases from young to middle adulthood [2-4], but the decrease levels off in old age $[1,5,6]$. However, the potential role of the gender differences in the affectivity trends through generations has been poorly explored. There is a paucity of data reporting the specific details of gender related changes in affect while people advance along the life span.

It was recently demonstrated that positive affect, as assessed by Global Mood Scale [7], moderates the effect of stress, and in turn, this stress reduction contributes to decreasing cardiovascular disease (CVD) risk [8]. For instance, an association was found between positive affect and lower 10-year incident CHD, suggesting that preventive strategies may be enhanced not only by reducing negative affect (i.e., depressive symptoms, anger, anxiety), but also by increasing positive affectivity [9] and self-esteem [10]. Hence, it could be expected that individuals with higher positive affect and lower negative affect may report or have better overall health. It was also found that patients with higher levels of positive affect were more likely to exercise [11]. Other factors can be mediating direction and rate of changes of affectivity over life-span. Netherlands Study of Depression and Anxiety [12] found that number of chronic disease is a risk factor of depression. Understanding the trends and characteristics of the time course development of affectivity over life-span could lead to targeted community and behavioral medicine programs tailored to the necessities of the aging population.
Accordingly, the aim of the present study was twofold: (i) examine positive and negative affectivity, its rate of change, and gender specificity through generations, and (ii) to explore the association between health self-rating, number of chronic diseases (morbidity), memory condition, mental stress in regards to both aspects (positive and negative) of affectivity.

\section{Method}

\section{Study design}

Data was obtained from the Longitudinal Study of Generation [13]. Freely available, anonymous and unidentified coded data were used. Data are available for download at The Inter-University Consortium for Political and Social Science of the Institute for Social Science at the University of Michigan.

In 1971, three generations of families were randomly chosen from health maintenance organizations (HMO) subscriber list in Southern California. Participants were assessed in 1971, 1985, 1988, 1991, 1994 and 1997, and 2000. There were a total of 2024 (57\% female) participants

*Corresponding author: Aleksandr Kaipov, Department of Psychiatry, Larkin Community Hospital, South Miami, FL 33143, USA, Tel: (305) 458-5098; Fax: (305) 284-7545; E-mail: akaipov@larkinhospital.com.

Received: January 25, 2018; Accepted: March 22, 2018; Published: April 10 2018

Citation: Kaipov A, Sanchez-Gonzalez MA, May RW, Dumenigo R, Oms JD (2018) Life Span Changes in Affectivity and Influence of Gender, Health Self-rate, Morbidity, Memory and Mental Stress. J Psychiatry 21: 446. doi:10.4172/2378 5756.1000446

Copyright: (C) 2018 Kaipov A, et al. This is an open-access article distributed under the terms of the Creative Commons Attribution License, which permits unrestricted use, distribution, and reproduction in any medium, provided the original author and source are credited 
in the sample. The age of participants varied from 16 (initial observation in 1971) to 99 years of age at 2000 .

Data on study variables (positive affect and self-rate of health) were available for a total of 1255 individuals in 1985, 1411 persons in 1988, 1452 individuals in 1991, 1623 individuals in 1994, 1677 individuals in 1997 and 1322 individuals in 2000.

\section{Variables and scales}

Gender: Gender was dummy coded and used in the growth curve model to examine whether gender is a factor to influence life-span trajectory of affectivity.

Affectivity: Affect was assessed with the Affect Balance Scale [14], with five items measuring positive affect and five items measuring negative affect. The validity of the scale has been repeatedly supported $[15,16]$. Maximal possible value for positive affect was 5 (higher scores reflect more positive affect), and 5 for negative affect (lower scores reflect less positive affect). Six waves of this parameter were included in analysis $(1985,1988,1991,1994,1997,2000)$.

Health self-rate: In the original study participants rated their health on a single item: "Compared to people of your own age, how would you rate your overall physical health at the present time?" Responses were measured on a 4 point scale ( $1=$ poor; $2=$ fair; 3 =good; $4=$ excellent). Six waves of this parameter were included in analysis $(1985,1988,1991$, 1994, 1997, and 2000).

Comorbidities: Participants were asked questions whether in the past years they had angina, high blood pressure, stroke, cancer, respiratory ailments, digestive problems, rheumatism, diabetes, orthopedic problems or injury, cataract, glaucoma. All answers (0-no, 1 -yes) were summarized and a new variable "morbidity" was calculated for every wave of study (total 6 waves: 1985, 1988, 1991, 1994, 1997, and 2000).

Memory: Memory was assessed by the question: "In general, would you say that your memory for day-to-day things is better than ever (1), as good as ever (2), is somewhat worse, (3) much worse or (4) now than it was a few years ago?" Four waves $(1985,1988,1997$, and 2000) of this parameter were available and included in analysis.

Mental distress: Mental distress was assessed by the question: "Have you had this health problem in last few years: severe mental distress?" with answers 0 (no) and 1 (yes). Three waves (1991, 1994, and 1997) of this parameter were available and included in analysis.

Longitudinal data analysis was used with calculation of linear growth model equations and latent growth curve model [17]. A linear growth model with time as the predictor was run to evaluate change of positive affect in generations and individual variability in initial level and the rate of change. A linear growth model with gender as the predictor evaluated whether males and females have different affect, and whether affectivity can be predicted by gender. A linear growth model was created with outcome (affect) at different times controlling for different predictors: self-rate health, morbidity, memory and mental stress. Mplus version 7.3 statistical package was used [18,19].

Models' average intercept with its variance, average linear slope with its variance and covariance between intercept and slope were interpreted. The model with intercept only was used as null model for other models to compare with and to calculate comparative fit index (CFI).CFI was used for comparison, though with caution, because the lack of correlations between variables and lack of centrality, considering the nature of longitudinal observation [20]. Likelihood ratio test was used to compare models and to assess whether more complex models changed model fit. In order to choose the preferred model, chi square should have been higher than critical values for calculated degree of freedom. Chi-squared test for model fit, root mean squared error of approximation (RMSEA) and standardized root mean square residual (SRMR) test were used to assess how well the model re-created the observed covariance matrix. Good fit of the model was considered with CFI values more than 0.95 and RMSEA values less than or equal 0.06 [16]. The average intercept was interpreted as the average value of outcome at time $=0$, controlling for predictor (gender, self-rate of health, chronic diseases co-morbidities, memory, mental distress). The average linear slope was interpreted as the average increase (or decrease) in the outcome for a 1-unit increase in time, controlling for the predictor. Positive covariance was interpreted as respondents with a high intercept having high slope and respondents with a low intercept having low slope. Negative covariance was interpreted as respondents with high intercept having low slope and respondents with low intercept having high slope.

\section{Results}

\section{Positive and negative affect}

The fit of the linear growth models was found to be overall satisfactory. CFI values ranged from 0.943 to 0.988 and RMSEA values ranged from 0.029 to 0.065 . The average value of positive affect in 1985 in the linear model was 4.280 . The average change of this outcome was expressed by negative slope of 0.118 . In other words, positive affect had a tendency to decrease every three years by 0.118 . The intercept and slope had positive significant covariance $(0.078, \mathrm{p}<0.001)$, suggesting that people with higher affect had a greater decrease through the generations.

The average value of negative affect in 1985 in the linear model was 2.146 . The average change of negative affect through generations was expressed by a negative slope of $0.098(\mathrm{p}<0.001)$. In other words, negative affect had a tendency to decrease every three years by 0.098 . Although small, the decrease in negative affect was significant and had negative significant covariance $(-0.097, \mathrm{p}<0.001)$, suggesting that people with higher negative affect had a lower decrease of it through generations. Taken together these results suggest that with time high positive affect has a tendency to decrease (positive covariance), while higher negative affect tends to persist (negative covariance) (Figure 1).

\section{Positive affects controlling for genders}

No gender differences either in intercept or in slope were found (Figure 2).

Males positive affect $=4.821-(0.275) \times($ time $)$

Females positive affect $=4.718^{\mathrm{NS}}-\left(0.265^{\mathrm{NS}}\right) \times($ time $)$

\section{Negative affects controlling for genders}

There was no difference in intercept value with significant positive difference in slope for females (Figure 3).

Males negative affect $=2.065-(0.251) \times($ time $)$

Females negative affect $=\left(1.966^{\mathrm{NS}}-\left(0.325^{\star *}\right) \times(\right.$ time $)$

In other words (Figure 4), positive affect had (1) no significant difference in initial average value in both sexes, (2) had a tendency to decrease in both male and female while they advance through the 
life span, and (3) with no difference in genders. Negative affect also was (1) not significantly different in both sexes' initial values, and (2) was decreasing in both males and females; and this decrease (3) was significantly higher in females.

\section{Health self-rate}

Health self-rates significantly increased with life progression, as demonstrated by significant positive slope $(0.033, \mathrm{p}<0.01)$. There was statistically significant negative covariance between intercept and slope $(-0.014, \mathrm{p}<0.001)$. Negative covariance indicates that individuals with high health self-rate display low slope, which means their high self-rate tends to persist while they age. There were no statistical differences between genders (Figure 5).

Males health $=1.872+(0.024) \times($ time $)$.

Females health self-rate $=1.848^{\mathrm{NS}}+\left(0.038^{\mathrm{NS}}\right) \times($ time $)$

\section{Morbidity}

Initially only 1242 out of 3496 participants fully answered questions about chronic diseases. 37\% marked number of chronic conditions " 0 ", $28 \%$ as " 1 ", $18.7 \%$ as " 2 ", and only $14 \%$ indicated number of chronic condition " 3 " and higher. Average value of morbidity at time $=0$ in 1991 for males (group coded one) respondents was 1.122 for intercept and non-significant positive 0.115 slope. No difference between genders was found (Figure 6).

Males morbidity $=1.122+(0.115) \times($ time $)$

Females morbidity $=1.187^{\mathrm{NS}}+\left(0.197^{\mathrm{NS}}\right) \times($ time $)$

\section{Memory}

Linear model revealed positive non-significant slope, indicating no change of reported memory along life span. No difference between genders was found (Figure 6).

$$
\begin{aligned}
& \text { Males memory }=2.548-(0.042) \times(\text { time }) \\
& \text { Females memory }=2.465^{\mathrm{NS}}-\left(0.004^{\mathrm{NS}}\right) \times(\text { time })
\end{aligned}
$$

\section{Mental stress}

Model revealed positive non-significant slope in general population with no respect to age, indicating no changes in reported stress along life span, although stress reported by female was higher at baseline (Figure 6).

$$
\begin{aligned}
& \text { Males mental stress }=0.001+0.022 \times(\text { time }) \\
& \text { Females memory }=0.033^{* * *}+\left(0.035^{\mathrm{NS}}\right) \times(\text { time })
\end{aligned}
$$

\section{Positive and negative affect controlling for different time invariant predictors}

In order to understand whether population affect can be predicted by such factors as health self-rate, morbidity, memory and mental stress, we recoded above variables to create two groups in each wave of observation.

\section{Health self-rate}

- Group 1: rated excellent (answer 1) or good (answer 2).

- Group 2: rated as fair (answer 3) or poor (answer 4).

\section{Morbidities}

- Group 1: number of chronic diseases 0 and 1.
- Group 2: chronic conditions 2 and more.

\section{Memory}

Group 1: rated memory as better (answer 1) or good (answer 2).

Group 2: rated memory as worst (answer 3) and much worst (answer 4).

Mental stress was binary variable initially, no recoding was needed. Group 1 consisted of participants with no stress and Group 2 with mental stress. The same linear model was applied to explore whether above binary factors at any time of the observation can predict level and change of positive and negative affect. In this model health, morbidity, memory and mental stress were used by exactly same way as gender, when male and female difference in the intercept and slope were calculated.

\section{Positive and negative affect controlling for health self-rat}

There was no differences in positive affect between groups 1 and 2 a time $=0$, but significant negative difference in slope.

Positive affect good health $=4.756-(0.079) \times($ time $)$

Positive affect poor health $=4.881^{\mathrm{NS}}-\left(0.305^{\star * *}\right) \times($ time $)$

Positive affect did not differ at baseline in groups with good and poor health, but decreased over life span with higher rate in people who initially rated their health as poor.

Negative affect at time $=0$ for group 2 was significantly higher than group 1. At the same time group 2 had significant negative slope indicating more pronounced decrease of negative affect along life span.

Negative affect good health $=1.342-(0.113) \times($ time $)$

Negative affect poor health $=1.881^{* * *}-\left(0.145^{\star \star}\right) \times($ time $)$

In other words, negative affect was significantly higher in people with poor health, but it revealed tendency to decrease over time (Figure 7).

\section{Positive and negative affect controlling for morbidity}

For people with higher morbidities, there was significant negative difference in intercept and non-significant positive difference in slope (Figure 8).

Positive affect low morbidity $=4.658-(0.155) \times($ time $)$

Positive affect high morbidity $=\left(4.352^{\star * \star}\right)-\left(0.145^{\mathrm{NS}}\right) \times($ time $)$

In other words people with higher morbidity at time $=0$ do have decreased positive affect, but morbidity is not a significant factor influencing positive affect changes over generations.

No significant association between negative affect and morbidities was found in both intercept and slop.

Negative affect low morbidity $=2.070-(0.155) \times($ time $)$

Negative affect high morbidity $=1.955^{\mathrm{NS}}-\left(0.097^{\mathrm{NS}}\right) \times($ time $)$

\section{Positive and negative affect controlling for memory}

No difference in positive affect at baseline at time $=0$ between people with good and bad memory noted. Worsening memory was associated with more pronounced drop of positive affect at aging, as evident by significant negative slope (Figure 9).

Positive affect good memory $=5.020-(0.233) \times($ time $)$ 


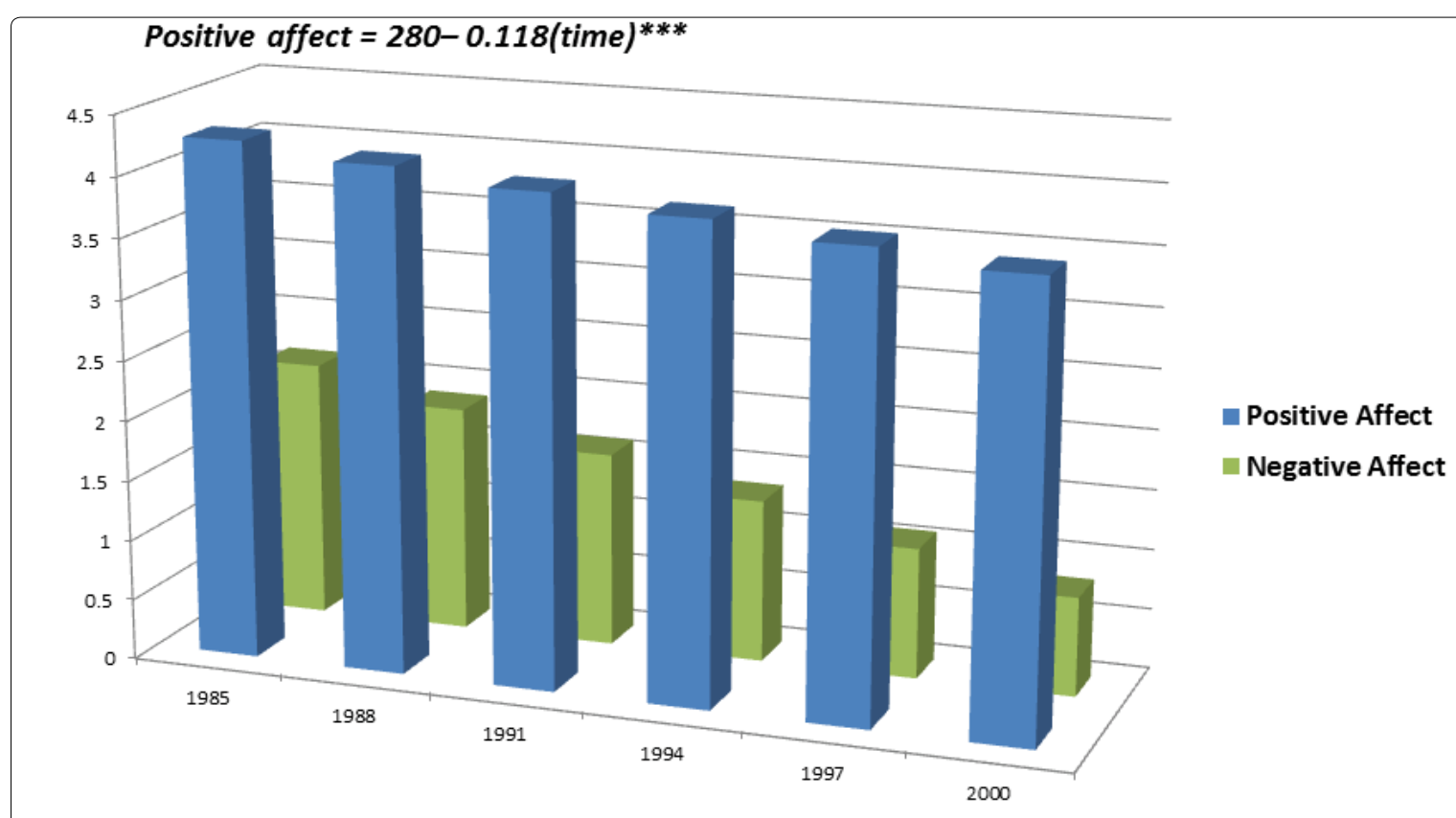

Negative Affect $=2.146-0.265$ (time) $* * *$

Figure 1: Changes in positive and negative affect through lifespan.

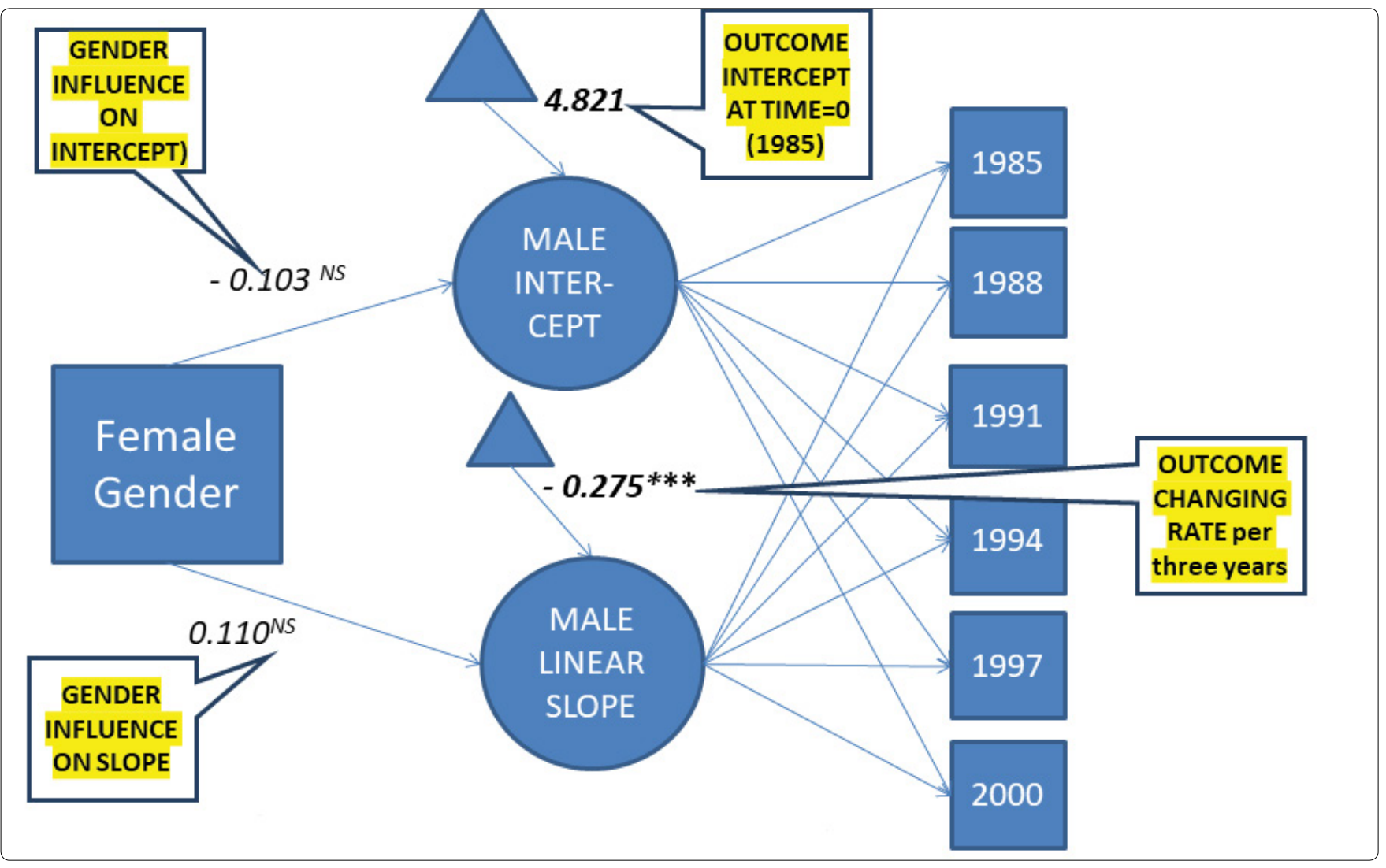

Note: NS-Not significant; ${ }^{*}-<0.05 ;{ }^{* *}-<0.001 ;{ }^{* * *}-<0.001$

Figure 2: Positive affect (outcome) controlling for gender. 


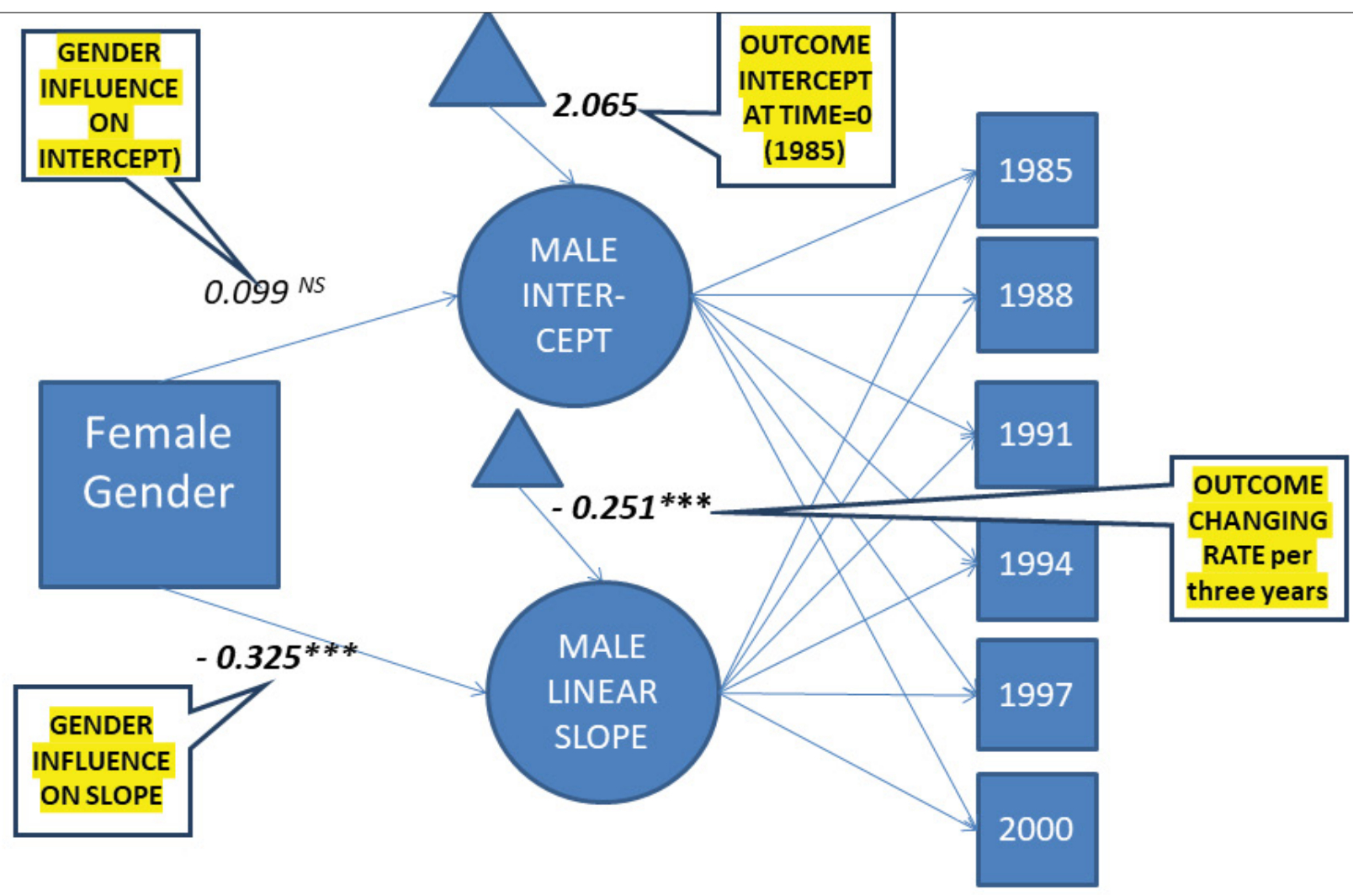

Note: NS-Not significant; ${ }^{*}-<0.05 ;{ }^{* *}-<0.001 ;{ }^{* * *}-<0.001$

Figure 3: Positive affect (outcome) controlling for gender.

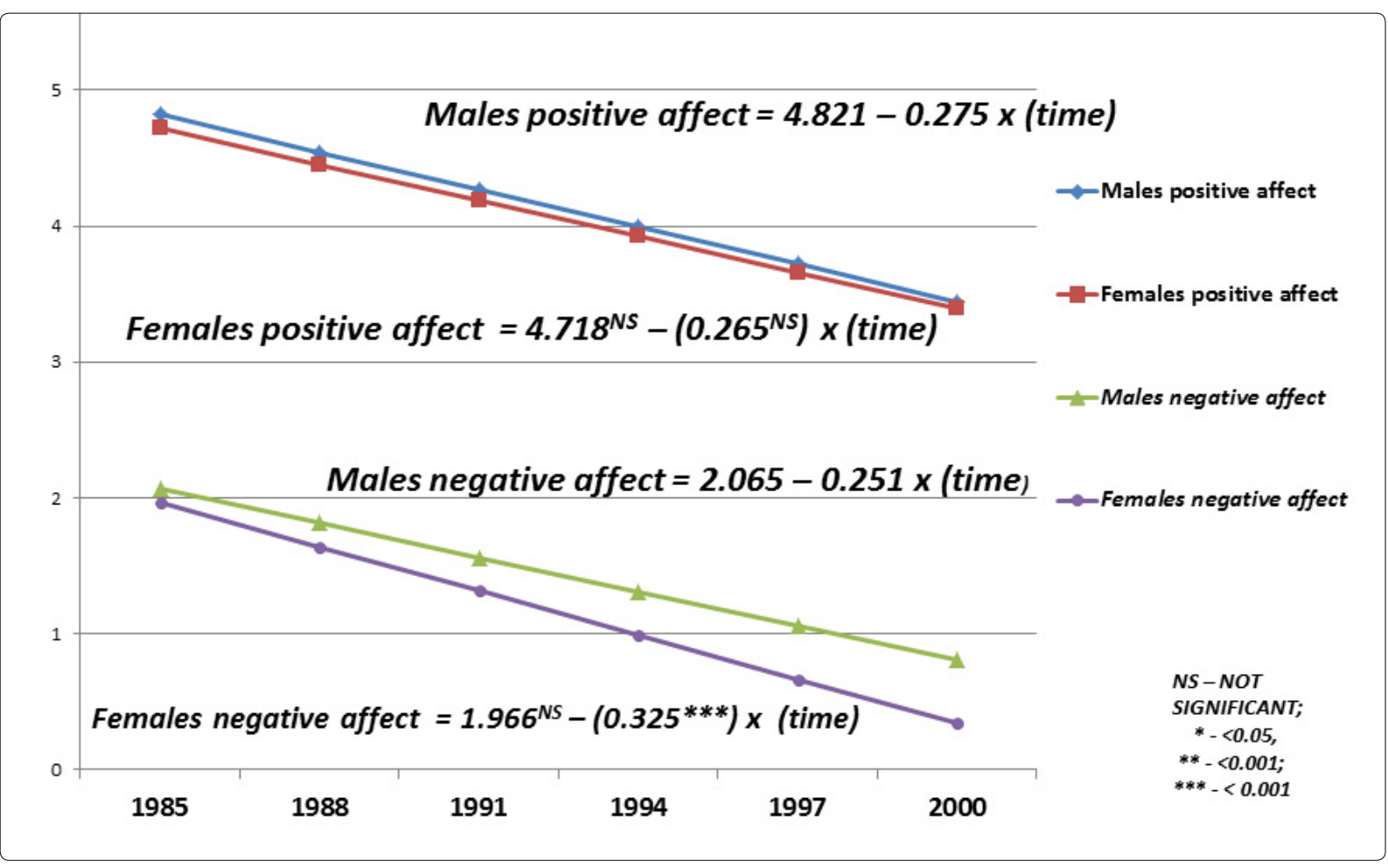

Figure 4: Positive and negative affect controlling for gender. 
Citation: Kaipov A, Sanchez-Gonzalez MA, May RW, Dumenigo R, Oms JD (2018) Life Span Changes in Affectivity and Influence of Gender, Health Self-rate, Morbidity, Memory and Mental Stress. J Psychiatry 21: 446. doi:10.4172/2378-5756.1000446

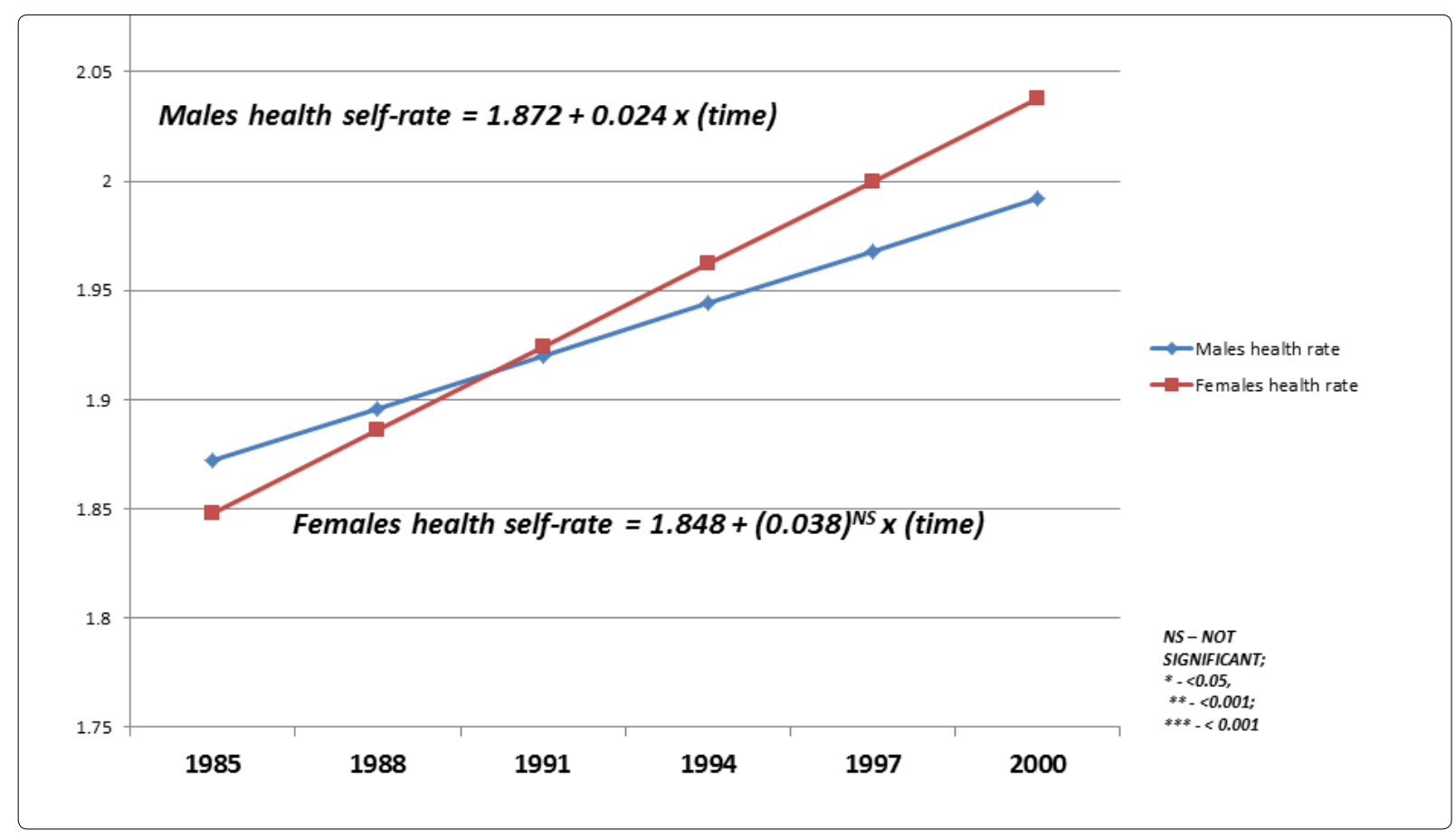

Figure 5: Health self-rate controlling for gender.

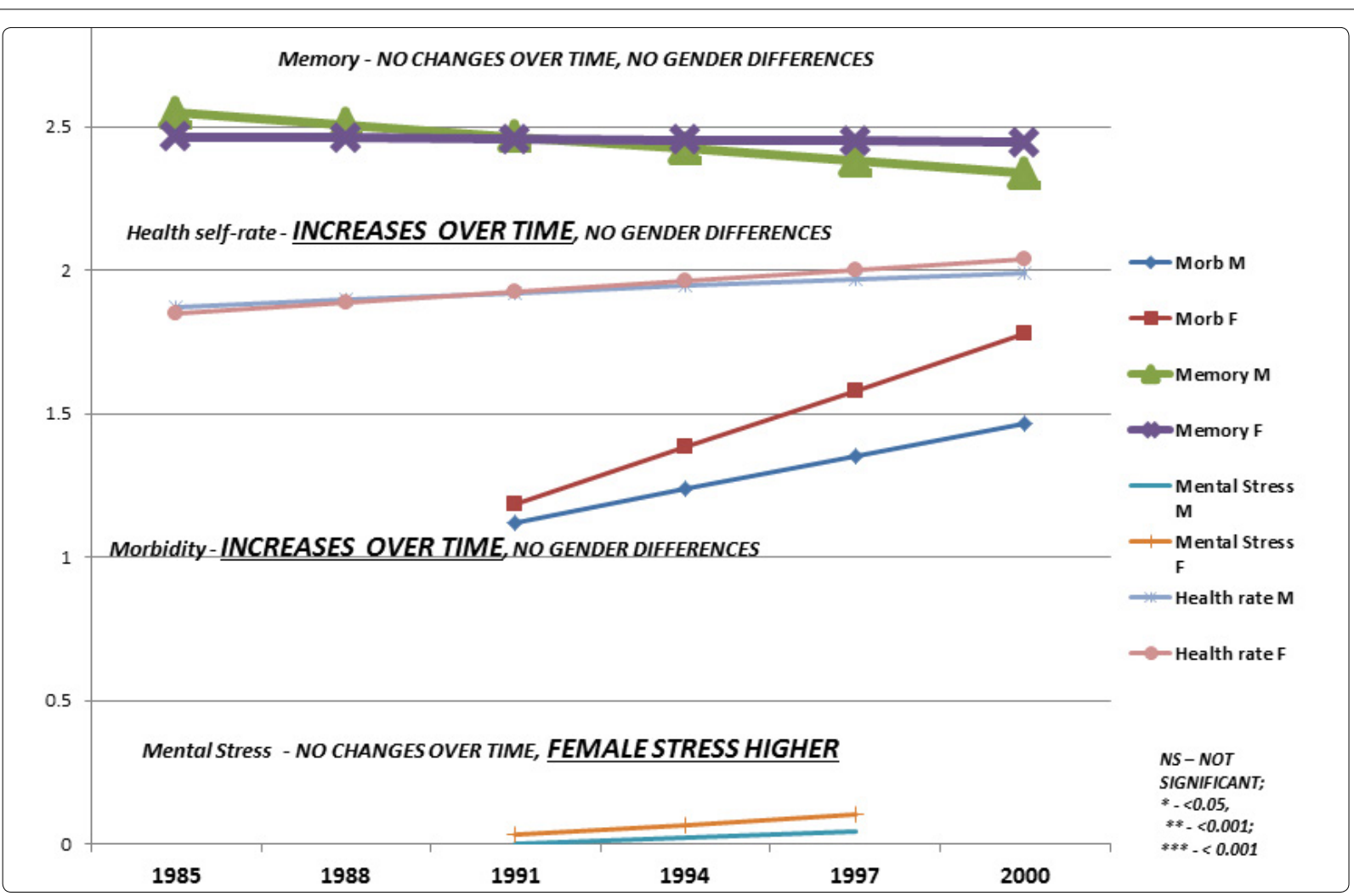

Figure 6: Memory, health self-rate, morbidity and mental stress controlling for gender.

Positive affect worst memory $=\left(4.973^{\mathrm{NS}}\right)-\left(0.344^{\star *}\right) \times($ time $)$

No difference was found between people with good or bad memory in both intercept and slope of negative affect.
Negative affect good memory $=(1.159)-(0.150) \times($ time $)$

Negative affect worst memory $=\left(1.319^{\mathrm{NS}}\right)-\left(0.120^{\mathrm{NS}}\right) \times($ time $)$ 


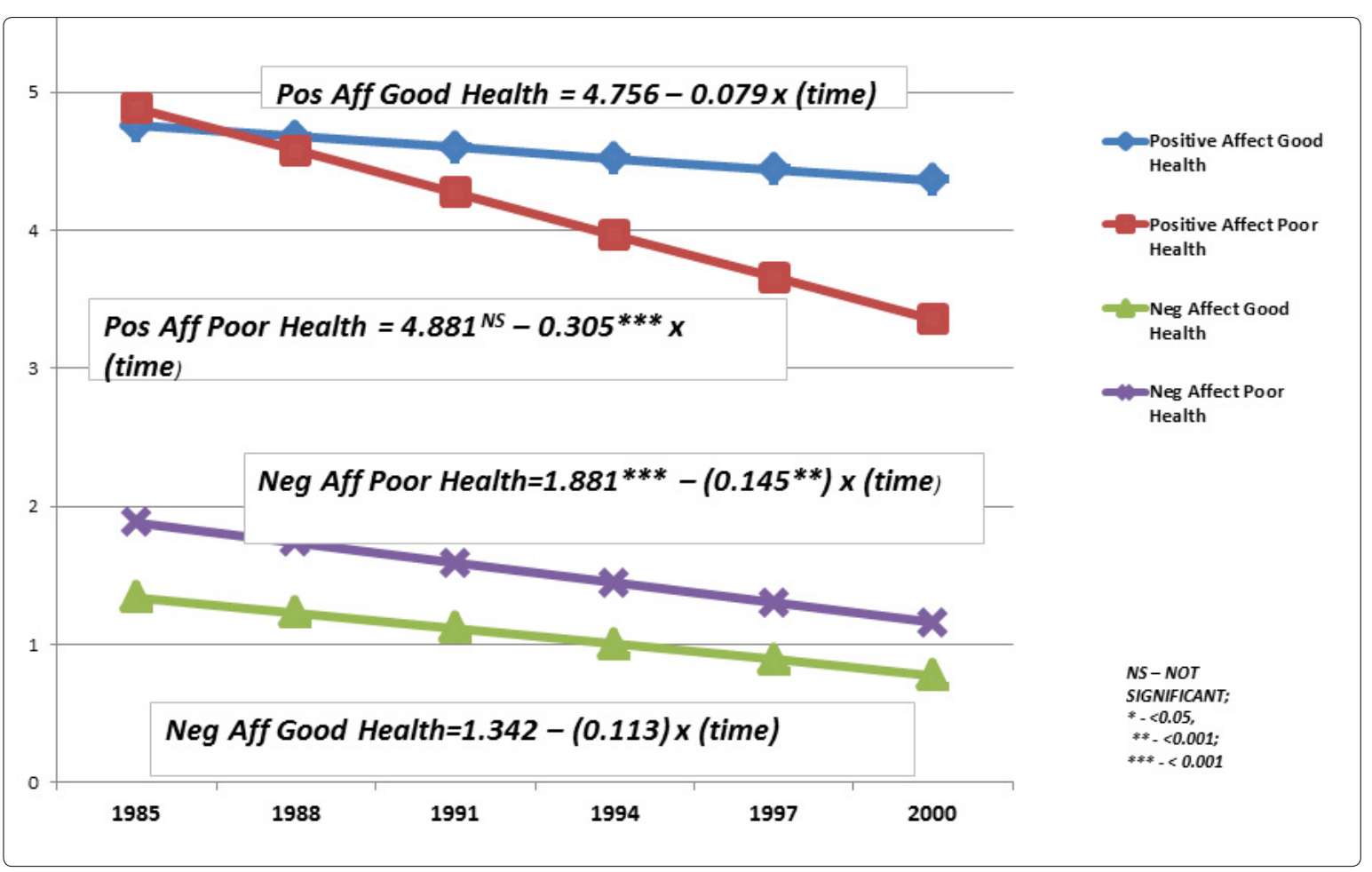

Figure 7: Positive and negative affect controlling for self-health rate.

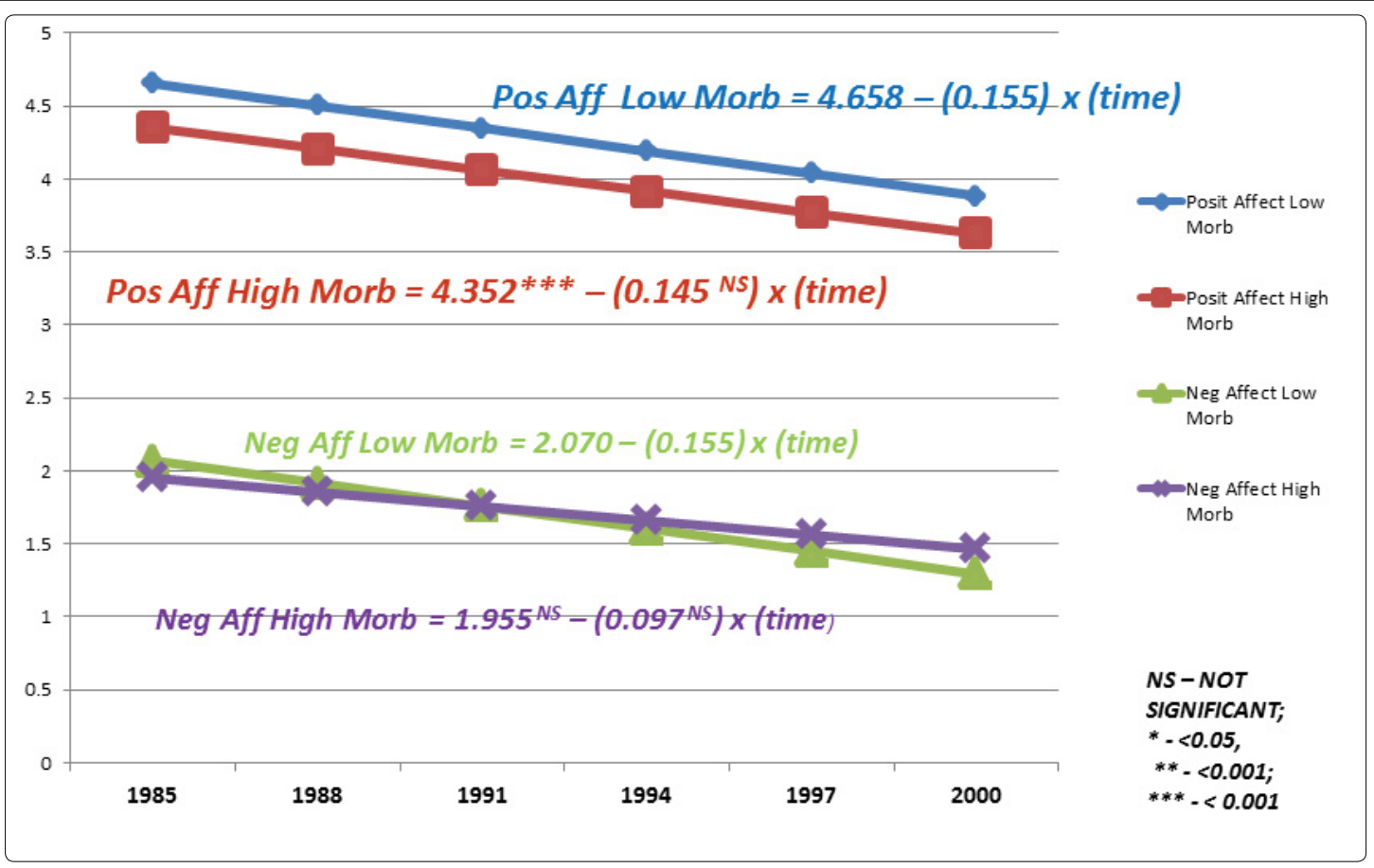

Figure 8: Positive and negative affect controlling for Morbidity.

\section{Positive and negative affect controlling for mental stress}

Positive affect was significantly lower at baseline in group with mental stress in history, though changes over time did not show dependency of effect on stress, evident by non-significant negative difference in slope (Figure 10).
Positive affect no stress $=4.279-(0.141) \times($ time $)$

Positive affect stress $=3.890^{*}-\left(0.163^{\mathrm{NS}}\right) \times($ time $)$

Negative affect was significantly higher in people with mental stress at time zero, but overall decrease of negative affect was not different 
Citation: Kaipov A, Sanchez-Gonzalez MA, May RW, Dumenigo R, Oms JD (2018) Life Span Changes in Affectivity and Influence of Gender, Health Self-rate, Morbidity, Memory and Mental Stress. J Psychiatry 21: 446. doi:10.4172/2378-5756.1000446

Page 8 of 10

in both groups as manifested by non-significant negative difference in slope.

Negative affect no stress $=1.842-(0.127) \times($ time $)$

Negative affect stress $=3.265^{\star * *}-\left(0.226^{\mathrm{NS}}\right) \times($ time $)$

\section{Discussion}

Life-span trajectory of affectivity was analyzed in previous study [10], but present on is the first study to our knowledge to explore gender specificity, and also novel in term of analyzing in relation to important life outcomes, specifically to health safe rate, morbidity, memory and mental stress, and their co-influence into the dynamics of life attitude, which is perceived and expressed by affect.

In this study we found that positive affect had no significant difference in initial average value between sexes, it had a tendency to decrease in generations and such decrease was similar in both genders. Negative effect also did not reveal gender differences at initial time, and also revealed tendency to decrease. It seems that both males and females demonstrate lesser affectivity along life span progression. But unlike positive affect, decrease in negative affect was significantly more pronounced in female individuals. These finding seem to point towards the conclusion that people have a tendency of lesser affectivity while growing older, and that females had no difference with males in positive affect changes, but revealed less negativity while growing older. $\mathrm{He}$ gender differences in affectivity decline may have potential clinical and behavioral implications.

In this study, we deliberately compared analysis of six waves and five waves of affect and received similar results, which were different only in terms of $\mathrm{p}$-value, but still remained significant. This finding by itself has been reported previously for positive affect [5] and negative affect [2-4]. The present analysis is novel as it unveiled that gender is predictive factor of future affect changes specifically in negative affect.
It looks like female gender is associated with less negativity while people growing older. Previously not reported and rather unusual finding was the fact that both males and females manifested tendency to self-rate their health higher when they advance along the lifespan. It could be speculated why people are more optimistic, while they should theoretically have more health problems, but having in mind lesser affectivity it becomes evident that emotional attitude may be involved in this perception, thus warranting more investigation in cognition science.

In order to explore more the above theoretical assumption we analyzed reported chronic conditions and found that number of such conditions went up along the aging progression with no difference between males and females. It is unclear whether people with multiple chronic conditions dropped off the study, were not aware of them, really do not have any or chosen not to report them, but one conclusion can be made: somatic morbidities have not much to do with affectivity and subjective assessment of health. This effect is observed with no respect to gender.

Analysis of memory revealed the fact that most people have a tendency to assess their memory as "worse than before" and this perception was not changing over time. Moreover, negative significant covariance between intercept and slope can be interpreted as people with worse memory reports lessen decrease over time, even though this decrease is not significant. It should be noted that even though morbidity increases over time with no difference in males and females, health rate self-assessment also increases in both genders. Once again, both males and females selfassess their health more positive when they age. Memory does not change over time with any difference in genders. Higher mental stress is associated with female gender, but did not change over time in both sexes. All of the above represent useful characteristics of population health, which could be used for planning of health intervention and targeting specific groups with appropriate goals.

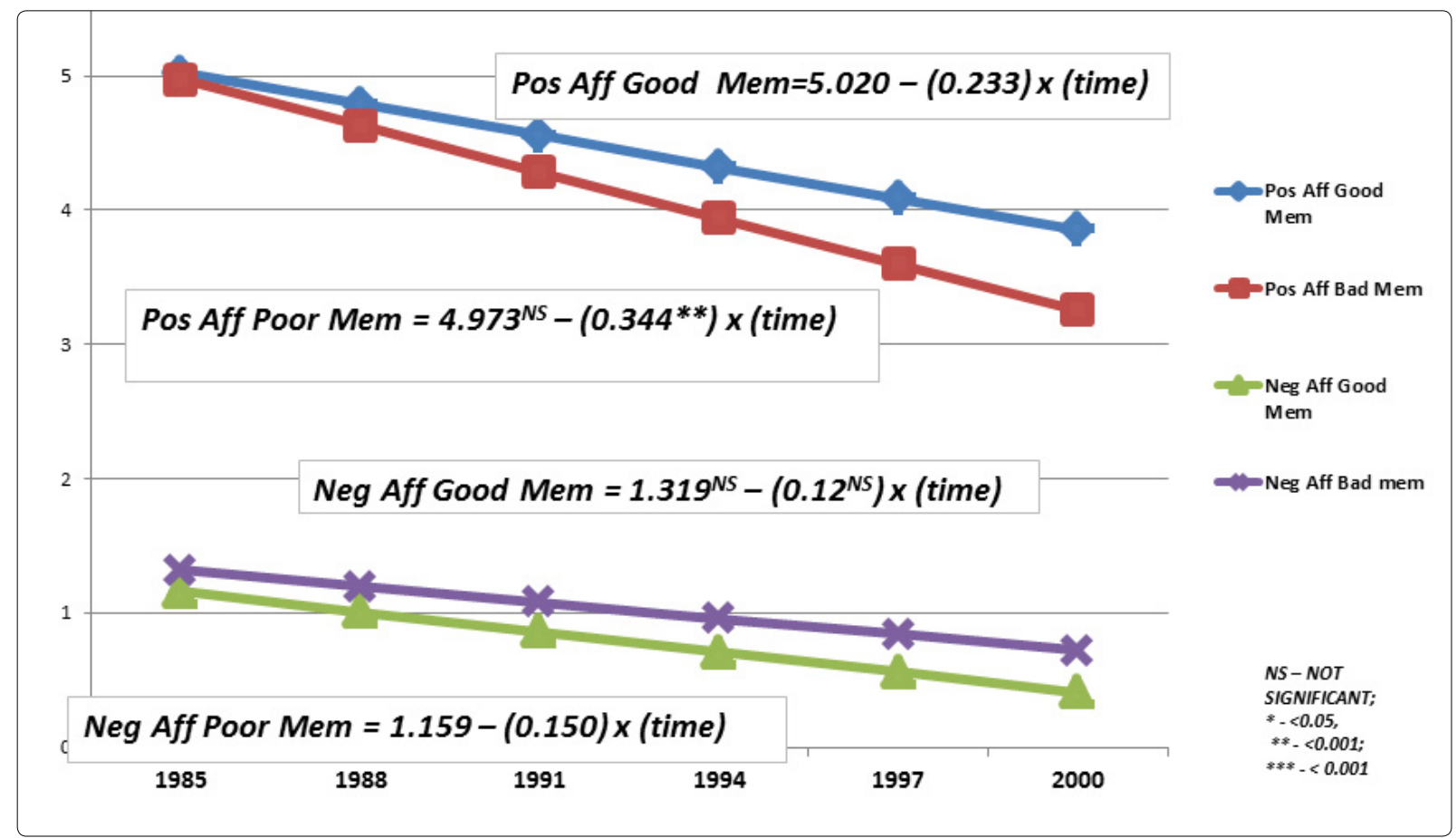

Figure 9: Positive and negative affect controlling for memory. 


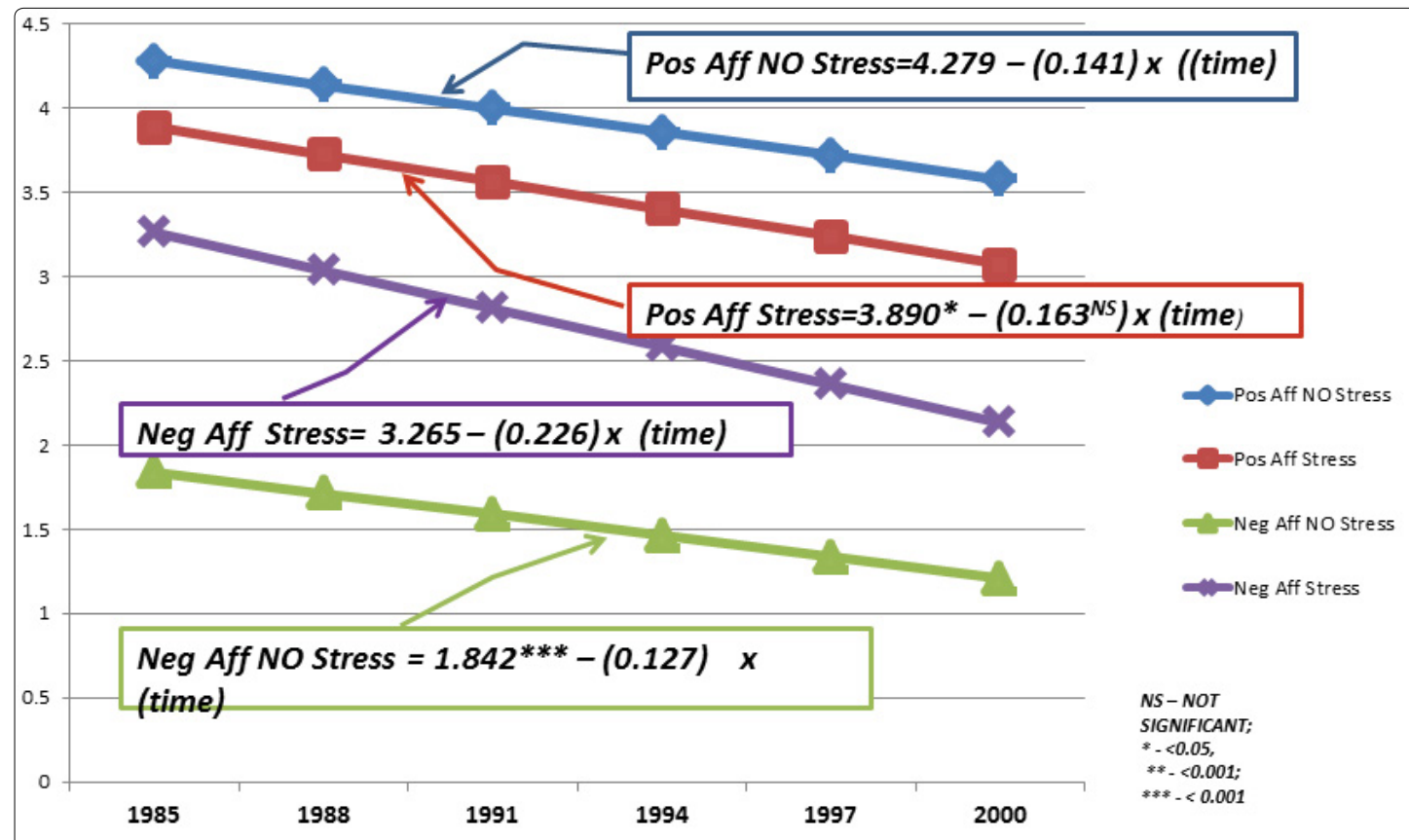

Figure 10: Positive and negative affect controlling for mental stress.

Higher morbidity index predicted lower positive affect at baseline, but morbidity was not found to be a significant factor influencing positive affect changes over generations. Morbidity was not a factor nor for intercept, do neither for slope of negative affect changes. It may mean that number of morbid conditions by itself is not the only factor which shapes and modulates affectivity. What such factors could be involved? Memory could be one of them, as worsening and bad memory was found to be a predictive factor of deeper drop of positive affect at aging. Mental stress is another potential factor, as positive affect was found to be significantly lower and negative affect significantly higher in group with mental stress in history, though changes over time did not show dependency of effect on stress.

Important limitations that should be considered in the interpretation of the analysis is that not all waves were available for comparison. Positive affect, negative affect and health self-rate were available in all six waves since 1985 through 2000. Memory, mental stress, and morbidities were available only partially in five, or only four waves. It does not change tendency, and does not make analysis results less reliable, but it makes it difficult to interpret tendency, as, for instance, no morbidities report was available in 1985 and 1988, so we have to use the morbidity starting from 1991 . The same is true about mental stress; first available set of data was collected in 1988. These limitations cannot be fixed, as all data have been collected long time ago, but should be taken into consideration.

The other limitation we have to mention is not really limitation, but rather, basis for further statistical workup. As we said before, longitudinal data analysis uses two types of predictors: time invariant and time varying. In the presented analysis we used time-invariant predictors only. They do not predict all growth parameters, but allows establishing baseline. For the future we will use not only time invariant predictors, but time varying and parallel processes for deeper exploration of affectivity.

\section{Conclusion}

- Both positive and negative affect decrease with age.

- Gender is time-invariant predicting factor for negative, but not for positive affect.

- Health self-rate increases over generations.

- Lower health self-rate is predictive factor for higher negative affected and associated with deeper drop of positive affect in generations.

- Morbidity or number of chronic conditions is a predictor of lower positive affect, but not for its changes over time.

- Morbidity is not a predictive factor for negative affect.

- Memory worsening is associated with decrease of positive affect over generations, and is not a predictive factor for negative affect.

- Mental stress is a predictive factor for lower positive and higher negative affect, but not predictor for their changes over time.

- The only predictors of future changes in affectivity are the lower health self-rate for both positive and negative affect and worsening memory for positive affect.

- Future analysis should include time varying predictors and parallel processes.

\section{Disclaimer}

No commercial funding support used from no company. No human subjects were involved. Freely available, anonymous and coded data were used. Data are available for download at The Inter-University Consortium for Political and Social Science of the Institute for Social Science at the University of Michigan. 
Citation: Kaipov A, Sanchez-Gonzalez MA, May RW, Dumenigo R, Oms JD (2018) Life Span Changes in Affectivity and Influence of Gender, Health Self-rate, Morbidity, Memory and Mental Stress. J Psychiatry 21: 446. doi:10.4172/2378-5756.1000446

\section{References}

1. Charles ST, Reynolds CA, Gatz M (2001) Age related differences and change in positive and negative affect over 23 years. J Pers Soc Psychol 80: 136151.

2. Helson R, Soto CJ (2005) Up and down in middle age: Monotonic and nonmonotonic changes in roles, status, and personality. J Pers Soc Psychol 89: 194-204.

3. Kessler EM, Staudinger UM (2009) Affective experience in adulthood and old age: The role of affective arousal and perceived affect regulation. Psychol Aging 24: $349-362$

4. Löckenhoff CE, Costa PT, Lane RD (2008) Age differences in descriptions of emotional experiences in oneself and others. J Gerontol B Psychol Sci Soc Sci 63B: 92-99.

5. Kunzmann U, Little TD, Smith J (2000) Is age related stability of subjective wellbeing a paradox? Cross-sectional and longitudinal evidence from the Berlin Aging Study. Psychol Aging 15: 511-526.

6. Carstensen LL, Pasupathi M, Mayr U, Nesselroade JR (2000) Emotional experience in everyday life across the adult life span. J Pers Soc Psycho 79: 644-655.

7. Denollet J, De Vries J (2006) Positive and negative affect within the realm of depression, stress and fatigue: the two-factor distress model of the Global Mood Scale (GMS). J Affect Disord 91: 171-180.

8. Pitt B, Deldin PG (2010) Depression and cardiovascular disease: have a happy day-just smile! Eur Heart J 31: 1036-1037.

9. Davidson KW, Mostofsky E, Whang W (2010) Don't worry, be happy: positive affect and reduced 10-year incident coronary heart disease: The Canadian Nova Scotia Health Survey 2010. Eur Heart J 31: 1065-1070.
10. Orth U, Robins WR, Widaman, KF (2012) Life-span development of selfesteem and its effects on important life outcomes. J Pers Soc Psychol 102 1271-1288.

11. Hoogwegt MT, Versteeg $\mathrm{H}$, Hansen TB, Thygesen LC, Pedersen S, et a (2013) Exercise mediates the association between positive affect and 5-year mortality in patients with ischemic heart disease. Circulation: Cardiovascular Quality and Outcomes. 6: 559-566

12. Schaakxs R, Comijs HC, Van der Mast RC, Schoevers RA, Beekman ATF, et al (2017) Risk factors for depression: Differential across age? Am J Geriatr Psychiatry 25: 966-977.

13. Silverstein, Merril, Bengtson VL (2016) Longitudinal study of generations 1971, 1985, 1988, 1991, 1994, 1997, 2000, 2005. Ml: Inter-university Consortium for Political and Social Research.

14. Bradburn NM (1969) The structure of psychological wellbeing

15. Harding SD (1982) Psychological wellbeing in Great Britain: An evaluation of the Bradburn affect balance scale. Personality Individ Differ 3: 167-175.

16. Kim KA, Mueller DJ (2001) To balance or not to balance: Confirmatory factor analysis of the affect balance scale. J Happiness Stud 2: 289-306.

17. Newsom JT, Jones RN, Hofer SM (2012) Longitudinal data analysis: A practical guide for researchers in aging, health, and social sciences. Routledge, New York.

18. Muthén BO, Muthén LK, Asparouhov T (2016) Regression and mediation analysis using Mplus.

19. https://www.statmodel.com/features.shtml

20. Raykov T (2005) Bias-corrected estimation of non-centrality parameters of covariance structure models. Struct Equ Modeling 12: 120-129. 